\begin{tabular}{|c|l|}
\hline Title & Experimental demonstration of a three qubit quantum computation algorithm using a single photon and linear optics \\
\hline Author(s) & Takeuchi, Shigeki \\
\hline Citation & $\begin{array}{l}\text { Physical Review A, 62, 032301 } \\
\text { https://doi.org/10.1103/PhysRevA .62.032301 }\end{array}$ \\
\hline Issue Date & 2000-08-03 \\
\hline Doc URL & http://hdl.handle.net/2115/5557 \\
\hline Rights & Copyright $\odot 2000$ A merican Physical Society \\
\hline Type & article \\
\hline File Information & PRA 62-3.pdf \\
\hline
\end{tabular}

Instructions for use 


\title{
Experimental demonstration of a three-qubit quantum computation algorithm using a single photon and linear optics
}

\author{
Shigeki Takeuchi* \\ Mitsubishi Electric Corporation, Advanced Technology Research and Development Center, Amagasaki, Hyogo 661-8661, Japan
}

(Received 17 February 1999; published 3 August 2000)

\begin{abstract}
A quantum computer that gives us the result of a single quantum computation has been constructed. The quantum register was realized by modes and polarization of photons, and the unitary transformation was implemented with linear optics. For each quantum computation, the answer to the Deutsch Jozsa problem for any four-bit digit is given by a single-photon detection signal with a small error rate of less than $4 \%$.
\end{abstract}

PACS number(s): 03.67.Lx, 42.30.-d

\begin{abstract}
${ }^{1}$ Quantum computation is a new concept that utilizes quantum superposition states for ultrafast parallel processing [1-3]. Deutsch and Jozsa found that quantum computers can segregate arrays of digits exponentially faster than classical computers [4], and the discovery was followed by Shor's famous factoring algorithm [5]. There have been several proposals for the actual realization of quantum computers [6-8]. Demonstrations of a quantum logic gate using single quanta have been performed $[9,10]$. However, the demonstration of algorithms has not been performed using a single-quantum system.
\end{abstract}

Nuclear magnetic resonance quantum computation (NMR-QC) was invented as a promising idea to realize quantum algorithms. The nuclear spins of a molecule in solution were adopted as qubits. Each of the molecules in the solution works as an individual quantum computer. So far, only NMR-QC has played the role of a test bed for these algorithms [11-14]. However, this test bed has the weak point that the results are always given by an average over a huge number of quantum systems, so "projection measurement' phenomena cannot be demonstrated by NMR-QC.

Projection measurement plays an important role in quantum computation. For the Deutsch-Jozsa algorithm, the structure was carefully selected so that the answer can be given by a single quantum computation [15]. In this sense, the experiment performed by NMR [13] did not demonstrate the heart of the algorithm sufficiently. As is pointed out in Ref. [14], the famous scheme of quantum error correction [16] based on projection measurement cannot be tested by NMRQC.

In addition, the input qubits were prepared in "hot mixed states" in the NMR-QC experiments performed. Because of these two problems, there is a class of quantum algorithms that cannot be solved efficiently by NMR-QC [17].

Quantum computation using linear optics is an alternative important test bed for quantum computing. If we use a single photon for computation, the result is given not by an average but by a single quantum computation. In quantum algorithms, appropriate unitary transformations are applied to quantum registers. Reck et al. [18] found that linear optics

\footnotetext{
*Present address: RIES, Hokkaido University, Sapporo 060-0812, Japan.
}

can be used to realize any unitary transformation. A theoretical proposal for quantum computation using linear optics was given by Takeuchi [19], and later a similar idea was suggested independently [20].

Here we report an experimental demonstration of the Deutsch-Jozsa quantum computation algorithm using linear optics and a single photon. In the experiment, the initial state was pure and the answer is given by single-photon detection so that the key aspect of the Deutsch-Jozsa algorithm of obtaining the answer with a single quantum computation is fully demonstrated. The experiment is equivalent to three qubits, which is the largest size of today's quantum computers. Our results also imply that quantum computation using linear optics is as practical as NMR-QC.

First, let us introduce the problem of the Deutsch-Jozsa algorithm. Suppose we are given an array of $2 N$ digits. We call the arrays "even" when they include as many 1's as 0's (e.g., $\{1,0,1,0\}$ for $N=2$ ), and "uniform"' when they are filled with only '0's or '1's (e.g., $\{1,1,1,1\}$ ). The problem for the Deutsch-Jozsa algorithm is to find the correct answer between "the given array is not even" and "the given array is not uniform." When the array satisfies both cases, either of them can be the answer. A classical computer needs $N$ +1 steps in the worst case. However, a quantum computer can find the answer with $O(\log (N))$ steps [4]. ${ }^{1}$

Our quantum computer solves the problem with four-bit inputs, for which three qubits are required in the DeutschJozsa algorithm; two qubits are used as the address register and one as the accumulator for the given oracle. In our computer, four optical paths are used for the address register and the polarization of the photon is used for the accumulator. Our quantum computer is used as follows. First, the computer is initialized for the computation. Second, the oracle (a four-bit digit) is given to the computer and is converted to the appropriate voltage applied to the E/O (electro-optic) modulators in the system. Then we put a single photon with vertical polarization into the input port of this quantum computer, and observe the detector at the output port to find whether it detects the photon or not. If the photon is detected,

\footnotetext{
${ }^{1} O$ is the Landau symbol: $h(x)=O(g(x))$ means $h(x) / g(x)$ is bounded for $x \rightarrow \infty$.
} 


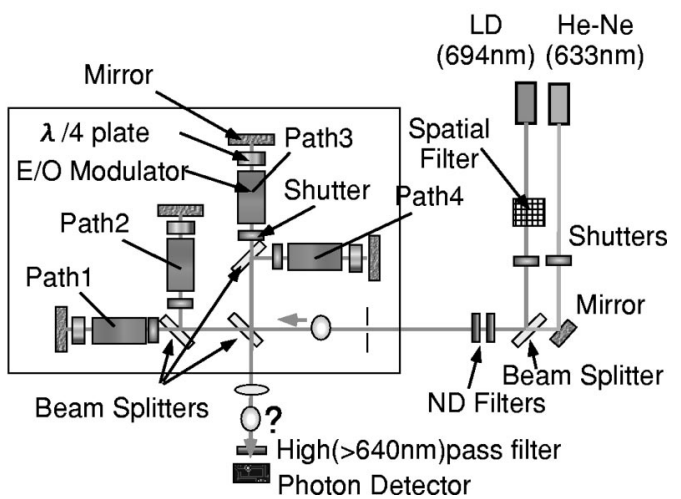

FIG. 1. Schematic diagram of the optical system for the Deutsch-Jozsa algorithm with four-bit inputs.

the answer is that the given oracle $\{f(j)\}$ is not even. If the photon is not detected, the answer is that the given oracle $\{f(j)\}$ is not uniform.

The experimental setup is shown in Fig. 1. The vertically polarized $694 \mathrm{~nm}$ beam from a laser diode passes through the spatial filter and is attenuated by neutral density (ND) filters to very weak light (up to $0.5 \mathrm{pW}$ ). When such a weak beam was used, the average number of photons present in the optical system was $3 \times 10^{-3}$, and the probability of finding two or more photons in the coherent length of $10 \mathrm{~cm}(\sim$ width of a single photon wave packet) was less than $3 \times 10^{-4}$. In addition, the number of incident photons was much less than the saturation level of the photon detector. In this sense, the computation was performed using the quantum phenomenon of single-photon interference. All shutters in the optical system except the one for the reference light were open during the computation.

The transformation of a 50:50 beam splitter is given as follows [21]:

$$
\frac{1}{\sqrt{2}}\left(\begin{array}{ll}
1 & i \\
i & 1
\end{array}\right) .
$$

After passing through three beam splitters, the wave function of a single photon is converted to a uniform superposition of the states passing through four optical paths. When the beam splitters are 50:50, the transformation of these three beam splitters is given using Eq. (1) as follows:

$$
\frac{1}{2}\left(\begin{array}{cccc}
1 & i \sqrt{2} & i & 0 \\
i & \sqrt{2} & -1 & 0 \\
i & 0 & 1 & i \sqrt{2} \\
-1 & 0 & i & \sqrt{2}
\end{array}\right) .
$$

Therefore, the wave function of the photon after passing these beam splitters can be described as follows [19]:

$$
I_{1}=\left[\begin{array}{l}
a_{1} \\
a_{2} \\
a_{3} \\
a_{4}
\end{array}\right][p]=\frac{1}{2}\left[\begin{array}{r}
1 \\
i \\
i \\
-1
\end{array}\right][0],
$$

where $a_{i}$ describes the amplitude of the wave function at path $i$ shown in Fig. 1, and $[p]$ describes the state of the polarization; [0] is the basis of the vertical polarization and [1] is that of the horizontal polarization. The phase factors according to the optical length of each path will be considered later. We use E/O modulators (Gsenger PM0202s) to embed the oracle $\{f(j)\}$ in the system. The modulators rotate the polarization of photons when and only when $f(j)=1$. After the modulation, the wave function becomes

$$
\begin{gathered}
\frac{1}{2}\left(\left[\begin{array}{l}
1 \\
0 \\
0 \\
0
\end{array}\right][f(1)]+i\left[\begin{array}{l}
0 \\
1 \\
0 \\
0
\end{array}\right][f(2)]+i\left[\begin{array}{l}
0 \\
0 \\
1 \\
0
\end{array}\right][f(3)]\right. \\
\left.-\left[\begin{array}{l}
0 \\
0 \\
0 \\
1
\end{array}\right][f(4)]\right)
\end{gathered}
$$

Next, quarter wave plates act as phase shifters, that change the phase of the wave function by $\pi / 2$ only when the polarization of the photons is vertical. After the phase shifters, the mirrors reflect back the wave function. The wave plates add another $\pi / 2$ phase shift according to the state of polarization. Then the E/O modulators rotate the polarization to the original state again. The state of the photon after the E/O modulators is written as follows:

$$
I_{2}=\frac{1}{2}\left[\begin{array}{r}
(-1)^{f(1)} \\
i(-1)^{f(2)} \\
i(-1)^{f(3)} \\
-(-1)^{f(4)}
\end{array}\right][0] .
$$

The transformation of three beam splitters can be written as follows:

$$
\frac{1}{2}\left(\begin{array}{cccc}
1 & i & i & -1 \\
i \sqrt{2} & \sqrt{2} & 0 & 0 \\
i & -1 & 1 & i \\
0 & 0 & i \sqrt{2} & \sqrt{2}
\end{array}\right) .
$$

After passing the three beam splitters again, the component $\psi_{3,[0]}$ of the wave function at output mode 3 with vertical polarization $([0])$ is written as

$$
\begin{aligned}
\psi_{3,[0]} & =i(-1)^{f(1)}-i(-1)^{f(2)}+i(-1)^{f(3)}-i(-1)^{f(4)} \\
& =\sum_{j=1}^{4}(-1)^{f(j)} \times \exp \left(i \phi_{j}\right)
\end{aligned}
$$

where $\phi_{j}$ is the phase corresponding to the optical path length of path $j$ and the additional phase factors due to the reflection at the beam splitters. 
Before the computation, we adjust the path length according to the following initialization procedure. Switching the shutters in the paths, and setting $f(1)=f(4)=1$ and $f(2)$ $=f(3)=0$, the visibilities of the interference between path 1 and path 3 , path 1 and path 2 , and path 3 and path 4 are observed sequentially and the tilt angles of the mirrors are adjusted to obtain the maximum visibilities, which were up to $98 \%$. Next, we control the length of path 1 to set the interferometers to a dark condition (almost no photons observed at the output port) with $0.5 \mathrm{~nm}$ precision using piezoactuators attached directly to each of the mirrors. Using Eq. 8 , the output of the interference between path 1 and path 3 is proportional to

$$
\begin{aligned}
& \left|(-1)^{f(1)} \exp \left(i \phi_{1}\right)+(-1)^{f(3)} \exp \left(i \phi_{3}\right)\right|^{2} \\
& \quad=2\left[1-\cos \left(\phi_{1}-\phi_{3}\right)\right] .
\end{aligned}
$$

Therefore setting the interferometer to the dark condition corresponds to having the condition $\phi_{1}=\phi_{3}$. In the same way, we realize the conditions $\phi_{1}=\phi_{2}$ and $\phi_{3}=\phi_{4}$ by setting the interference between path 1 and path 2 , and path 3 and path 4 , respectively, to the dark condition. The interference of the reference light $(632.8 \mathrm{~nm})$ was used for precise control of the path lengths. The optical system was also passively stabilized against thermal drifts for at least $10 \mathrm{~s}$ without active control.

Because the phase factors $\phi_{j}$ in Eq. (8) are set to be identical in the initialization procedure, the probability of the detection of the photon at the output port is

$$
P(\{f(j)\})=\frac{1}{16}\left|\sum_{j=1}^{4}(-1)^{f(j)}\right|^{2} .
$$

$P=0$ for the even input and 1 for the uniform input.

We used a single-photon counting module (SPCM-AQ, EG\&G) as the photon detection device. The observed dark count in the experimental setup was less than $4 \times 10^{3}$ counts/s.

For a given oracle, the photons were counted for $0.1 \mathrm{~s}$. By running the initialization procedure at intervals of $10 \mathrm{~s}$, we succeeded in continuing computation for tens of minutes. This initialization procedure was automatically performed by a personal computer, which also generates the four-bit digits and collects the data.

When the answer of our quantum computer is wrong for the given input, it is termed an "error." The error rate can be calculated from the photon detection probability $P(\{f(j)\})$ with the given oracle $\{f(j)\}$.

When we use weak light for the source of photons, we somehow have to know when a photon is not detected. One solution is to put three other photon detectors at the open output of the beam splitters. When we observe a photon detection signal from them, we regard the event as "not detected." In this experiment we just set the detector at the output port. In order to derive the detection probability

$$
P(\{f(j)\})=\frac{N(\{f(j)\})}{N_{\text {total }}},
$$

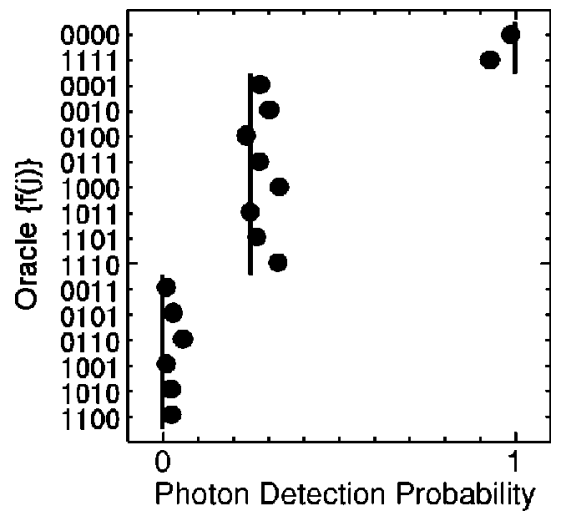

FIG. 2. The photon detection probability for the given four-bit digits $f(j)$. The theoretical values with no errors are shown by the solid lines, and the experimental values are plotted as black dots.

where $N(\{f(j)\})$ is the photon counting rate with the oracle $\{f(j)\}$, we have to estimate $N_{\text {total }}$, which is the number of total photon detection events of all detectors. In this paper, we estimated $N_{\text {total }}$ by $\left(N_{\max }+N_{\min }\right)$, where $N_{\max }$ is the maximum photon counting rate $\left(5.70 \times 10^{5}\right.$ counts/s) observed with oracles $\{f(j)\}=\{0,0,0,0\}$ and $N_{\text {min }}$ is the minimum counting rate $(6360$ counts/s) at $\{f(i)\}=\{1,0,0,1\}$. The experimental results are shown in Fig. 2. The vertical axis shows the four-bit digits given to the computer. The horizontal axis shows the probability $P$ of photon observation at the output port. The theoretical values given by Eq. (10) are shown by the solid lines in Fig. 2. The experimental values plotted as the black dots are calculated by Eq. (11).

This result shows that we can determine whether the statement "the given oracle $\{f(j)\}$ is not even" or "the given oracle $\{f(j)\}$ is not uniform" is correct with the small average error rates of $2.7 \%$ and $4.0 \%$, respectively, by the observation of a single-photon. Recently, we succeeded in analyzing the sources of errors quantitatively and found that the imperfection of the path length adjustment in the initialization procedure was the main cause. The subtle differences of the probabilities for intermediate cases (i.e., 1,0,1,1) from the theoretically predicted value of $25 \%$ seemed to come from the same source. The details of the analysis will be reported elsewhere [22].

We emphasize that the answer to the Deutsch-Jozsa problem was given here by a single quantum computation. The experiment thus exactly demonstrates the essence of the original Deutsch-Jozsa algorithm [15]. This is an important feature of linear optics quantum computation when compared with the NMR-QC method. The observation of a stop bit of the quantum computer [23] and the quantum error correcting codes [16] are examples in which the projection measurement plays an important role. Although the implementation using linear optics is not suitable for large-scale computation because it requires $2^{N-1}$ paths for the demonstration with $N$ qubits, we believe it will become an important testing ground for quantum computation.

Let us briefly discuss the number of calculation steps. In quantum computation, the number of steps is regarded as the number of unitary transformations applied to the quantum register. Hence we should count the number of layers of the 
optics that the photon wave function was actually affected by. In our system, the total number of steps was eight (two for initial beam splitting, four for $\mathrm{E} / \mathrm{O}$ modulators and wave plates for a round trip, and two for final beam splitting). It is also interesting that the calculation of a four-bit digit can be performed using only a single photon. It is not the number of particles, but the number of the degrees of freedom of the particles, that is important for computation. After the submission of this paper, an experiment using Glover's algo-

[1] D. Deutsch, Proc. R. Soc. London, Ser. A 400, 97 (1985).

[2] A. Ekert and R. Jozsa, Rev. Mod. Phys. 68, 733 (1996).

[3] D. P. Divincenzo, Science 270, 255 (1995).

[4] D. Deutsch and R. Jozsa, Proc. R. Soc. London, Ser. A 439, 553 (1992).

[5] P. W. Shor, in Proceedings of the 35th Annual Symposium on Foundations of Computer Science, edited by S. Goldwasser (IEEE Computer Society, Los Alamitos, CA, 1994), p. 124.

[6] A. Barenco, D. Deutsch, A. Ekert, and R. Jozsa, Phys. Rev. Lett. 74, 4083 (1995).

[7] J. I. Cirac and P. Zoller, Phys. Rev. Lett. 74, 4091 (1995).

[8] B. E. Kane, Nature (London) 393, 133 (1998).

[9] C. Monroe, D. M. Meekhof, B. E. King, W. M. Itano, and D. J. Wineland, Phys. Rev. Lett. 75, 4714 (1995).

[10] Q. A. Turchette, C. J. Hood, W. Lange, H. Mabuchi, and H. J. Kimble, Phys. Rev. Lett. 75, 4710 (1995).

[11] N. A. Gershenfeld and I. Chuang, Science 275, 350 (1997).

[12] D. G. Cory, M. D. Price, and T. F. Havel, Physica D 120, 82 (1998).

[13] N. Linden, H. Barjat, and R. Freeman, Chem. Phys. Lett. 296 61 (1998).

[14] D. Leung, L. Vandersypen, X. Zhou, M. Sherwood, C. Yannoni, M. Kubinec, and I. Chuang, Phys. Rev. A 60, 1924 rithm with two qubits and a proposed system of three qubits were reported independently [24].

I would like to thank Professor Oliver Wright and Dr. Toshiro Isu for their fruitful comments on this paper and the members of the Quantum Devices Team at ATRC for their help with the experiment. The author is affiliated with the PRESTO project of the Japan Science and Technology Corporation.

(1999).

[15] When we did not detect the photon, the only information we obtained was that the detection probability was not 1 . Deutsch and Jozsa set up their problem originally according to the information we can obtain with a single quantum computation.

[16] D. P. DiVincenzo and P. W. Shor, Phys. Rev. Lett. 77, 3260 (1998).

[17] X. Zhou, D. W. Leung, and I. L. Chuang, e-print quant-ph/9906112.

[18] M. Reck, A. Zeilinger, H. J. Bernstein, and P. Bertano, Phys. Rev. Lett. 73, 58 (1994).

[19] S. Takeuchi, in Proceedings of the Fourth Workshop on Physics and Computation: PhysComp96, edited by T. Toffoli (New England Complex Systems Institute, Boston, 1996), pp. 299302.

[20] N. J. Cerf, C. Adami, and P. G. Kwiat, Phys. Rev. A 57, R1477 (1998).

[21] D. F. Walls and G. J. Milburn, Quantum Optics (SpringerVerlag, Berlin, 1994), p. 274.

[22] S. Takeuchi, Phys. Rev. A 61, 052302 (2000).

[23] M. Ozawa, Phys. Rev. Lett. 80, 631 (1998).

[24] P. G. Kwiat, J. R. Mitchell, P. D. D. Schwindt, and A. G. White, e-print quant-ph/9905086. 